\title{
Modulatory Effects of Human Bone Marrow-Derived Mesenchymal Stem Cells on Proliferation and Ultrastructural Changes in K562 Leukemic Cells
}

\author{
Jun How Low ${ }^{1,2}$, Ammu Kutty Radhakrishnan ${ }^{1 *}$, Premdass Ramdas ${ }^{1}$ and Wong Chee Yin ${ }^{4}$ \\ ${ }^{1}$ Division of Pathology, School of Medicine, International Medical University, Malaysia \\ ${ }^{2}$ Caboolture Hospital, Caboolture, Queensland Australia \\ ${ }^{3}$ School of Health Sciences, International Medical University, Malaysia \\ ${ }^{4}$ Cytopeutics Sdn Bhd Selangor, Malaysia
}

Received: March 01, 2018; Published: March 15, 2018

*Corresponding author: Ammu Kutty Radhakrishnan, Professor (Pathology Division), School of Medicine, International Medical University, No. 126, Jalan Jalil Perkasa 19, Bukit Jalil, 57000 Kuala Lumpur, Malaysia, Tel: 60-3-27317205; Fax: 60-3-86567229; Email: ammu_radhakrishnan@imu.edu.my

\begin{abstract}
Mesenchymal stem cells (MSCs) have been reported to possess modulatory effects on various types of cancers as well as immunomodulatory properties, which have intrigued many researchers. Over the past two decades, there has been a focus in literature on the interaction between MSCs and leukemias. However, the interactions between the MSCs and leukemic cells, in particular the k562 cells, have not been well documented. The aim of this study was to demonstrate the interactions between human bone marrow-derived MSCs (hBM-MSCs) on a chronic myeloid leukemic cell line (K562 cells). Co-culture of K562 cells with the hBM-MSC increased the viability of these cells in noncontact and contact co-culture conditions. The same was observed when the K562 cells were cultured in hBM-MSC conditioned medium. Transmission electron microscopy (TEM) of K562 cells co-cultured with hBM-MSCs showed significant ultra-structural observations consistent with biochemical assays performed. The findings suggest that although cell-to-cell interaction is required for effective modulatory effect to occur, soluble substance secreted by the hBM-MSCs may also be able to emulate similar effects.
\end{abstract}

Keywords: Bone Marrow-Derived Mesenchymal Stem Cells (MSC); Chronic Myeloid Leukemia (CML); Mitochondria; Transmission Electron Microscopy (TEM)

Abbreviations: MSC: Bone Marrow-Derived Mesenchymal Stem Cells; CML: Chronic Myeloid Leukemia; TEM: Transmission Electron Microscopy; GVHD: Graft-Versus-Host Disease; FBS: Fetal Bovine Serum (FBS)

\section{Introduction}

Leukemia is a type of primary neoplastic disorder which originates from the bone marrow. Ever since its discovery in the $19^{\text {th }}$ century, numerous research endeavors have focused on the understanding behind the pathogenesis of leukemia as well as its treatment [1]. To date, hundreds of genetic aberrations have been attributed to the pathogenesis of leukemia [2]. Although our knowledge of its treatment and management has progressively increased over the years, even with today's advance medical technology, leukemia remains the $11^{\text {th }}$ most common cancer in the World [3]. Mortality resulting from the condition was reported to be as high as $73.4 \%$ [3]. Evidently, there is much room for improvement from our understanding towards the development of leukemia to our treatment and management approach for the disease.

Mesenchymal stem cells (MSCs) belong to a group of heterogeneous multipotent stem cells that have long been marveled for its therapeutic potential in many incapacitating diseases ever since its first identification [4]. Its potential to differentiate into multiple tissues has produced considerable interest among researchers, especially in the field of regenerative medicine [5]. In addition to its multi-potential capability, the MSCs have been reported to possess immunomodulatory properties [6]. Due to those properties, they are immune privileged cells which have considerable therapeutic values in autoimmune diseases such 
as organ transplants [7]. To date, numerous clinical studies have since incorporated MSC therapy in an attempt to treat pathological diseases, such as graft-versus-host disease (GVHD) [8-11]. There has been a growing body of research which report on the interactions between MSCs and various types of cancer $[12,13]$. However, the results and findings regarding their behavior towards cancer have been controversial. Some studies reported that MSCs may have the ability to induce cancer cell death in vitro [14] while other studies have documented that they may actually support tumor growth and metastasis [13].

In order to elucidate the pathophysiology of leukemia, it is important to understand the hematopoietic microenvironment of the bone marrow (BM), which plays a pivotal role in the development and evolution of leukemia. In the bone marrow microenvironment, the BM-derived MSCs (BM-MSCs) constitute a major component. Therefore, a greater understanding of interactions between the BM-MSCs and leukemic cells may provide insights on the dynamic interplay between these two entities. To date, several studies have reported that MSCs possessing anti-apoptotic effects on various subtypes of leukemia $[15,16]$. This protective modulatory effect that MSCs seems to exert in various other tumor cells and their effect on the growth and sustenance of the malignant processes has also been documented in several other studies $[13,17]$. The MSCs were also found to have the ability to arrest leukemic cell division [18]. Therefore, the mechanistic nature of the interactions between MSCs and leukemic cells remains to be further illustrated. This aim of this study is to validate the modulatory effects of human BM-derived MSCs (hBM-MSCs) on K562 chronic myeloid leukemic (CML) cells using biochemical assays and changes at the ultrastructural levels.

\section{Materials and Methods}

\section{Cell Lines}

The K562 CML cells are suspension cells and were cultured using RPMI medium (Mediatech, USA) supplemented with 10\% fetal bovine serum (FBS) (Sigma, USA), 1\% sodium pyruvate (Mediatech, USA), 1\% penicillin-streptomycin (Mediatech, USA) under conditions set at $37^{\circ} \mathrm{C}$ in a humidified $5 \% \mathrm{CO}_{2}$ atmosphere. The human BM-derived MSCs (hBM-MSCc) were a kind gift from Cytopeutics Sdn Bhd (Malaysia), given for the sole purpose of carrying out this study were cultured using low-glucose DMEM medium (Sigma, USA) supplemented with 10\% FBS, 1\% glutamax (Gibco, USA), $5 \mathrm{ng} / \mathrm{ml} \mathrm{bFGF} \mathrm{(Millipore,} \mathrm{USA),} 5 \mathrm{ng} / \mathrm{ml}$ EGF (Millipore, USA) and $1 \%$ antibiotic-antimycotic solution (Gibco, USA) at $37^{\circ} \mathrm{C}$ in a humidified $5 \% \mathrm{CO}_{2}$ atmosphere. For serum-free experiments, the hBM-MSCs were cultured using a serum-free medium (Invitrogen, USA) supplemented with $1 \%$ antibiotic-antimycotic solution (Gibco, USA). The culture was maintained similarly at $37^{\circ} \mathrm{C}$ in a humidified $5 \% \mathrm{CO}_{2}$ atmosphere.

\section{Co-culture with hBM-MSCs}

Various concentrations of the hBM-MSCs ( 0 to $2.5 \times 10^{4}$ cells / $\mathrm{ml}$ ) were seeded in the wells of a six-well plate. The hBM-MSCs were allowed to adhere to the plate overnight. The medium was then carefully removed replaced with fresh medium. To each test and control well, $1 \mathrm{ml}$ of the $\mathrm{K} 562$ cells $\left(2.5 \times 10^{5}\right.$ cells $\left./ \mathrm{ml}\right)$ was added. The hBM-MSCs to K562 ratio was maintained at $1: 10$ as previously described [33]. The cells were incubated at $37^{\circ} \mathrm{C}$ in a humidified $5 \% \mathrm{CO}_{2}$ atmosphere for 24, 48 and 72 hours. Co-culture with hBM-MSCs was also performed using specialized serum-free medium (Invitrogen, USA).

\section{K562 Cultured with Conditioned Medium from hBM-MSC}

The hBM-MSCs $\left(2.5 \times 10^{4}\right.$ cells $\left./ \mathrm{ml}\right)$ were seeded in the wells of a 6-well plate and left overnight at $37^{\circ} \mathrm{C}$ in a humidified $5 \% \mathrm{CO}_{2}$ atmosphere. The medium was carefully removed and replaced with fresh medium. The plate was returned to the incubator at $37^{\circ} \mathrm{C}$ in a humidified $5 \% \mathrm{CO}_{2}$ atmosphere to be conditioned for 24,48 and 72 hours. At each interval, the hBM-MSCs culture medium was carefully removed in to a tube and centrifuged (1500 rpm for 5 mins) to remove any detached cells. The conditioned medium was then added to the $\mathrm{K} 562$ cells $\left(2.5 \times 10^{5}\right.$ cells $\left./ \mathrm{ml}\right)$ freshly plated into a 6-well plate. Fresh unconditioned hBM-MSCs culture medium was added to the control well containing the K562 cells. The cultures were incubated at $37^{\circ} \mathrm{C}$ in a humidified $5 \% \mathrm{CO}_{2}$ atmosphere for 24 , 48 and 72 hours. Viability of the K562 cells was determined using the MTT [3-(4, 5-dimethylthiazol-2-yl)-2, 5-diphenyltetrazolium bromide] assay as described previously [19]. The MTT assay was performed using triplicate wells and the experiment was repeated twice to ensure reproducibility.

\section{Transwell (Non-contact) Culture with hBM-MSC}

Various concentrations ( 0 to $2.5 \times 10^{4}$ cells $/ \mathrm{ml}$ ) of hBM-MSCs were seeded in Transwell inserts (BD Biosciences, USA) fitted into the wells of a 6-well plate using a sterile forceps. The hBM-MSCs were allowed to adhere to the inserts overnight. The medium was then carefully removed and replaced with fresh new medium. The inserts were carefully removed using a sterile forceps to add $1 \mathrm{ml}$ of $\mathrm{K} 562$ cells $\left(2.5 \times 10^{5}\right.$ cells $\left./ \mathrm{ml}\right)$ into each test and control well, before fitting the inserts back into the wells. The cells were incubated at $37^{\circ} \mathrm{C}$ in a humidified $5 \% \mathrm{CO}_{2}$ atmosphere for 24,48 and 72 hours. Transwell inserts of $0.4 \mu \mathrm{m}$ and $1.0 \mu \mathrm{m}$ pore sizes were used. Viability of the K562 cells after the three culture intervals was determined using the MTT assay. The MTT assay was performed using triplicate wells and the experiment was repeated twice to ensure reproducibility.

\section{Ultrastructural Analysis of K562 Co-cultured with hBM- MSC using Transmission Electron Microscopy}

The K562 cells were subjected for transmission electron microscopy (TEM) to investigate the ultra-structural changes (if any) following co-culture with the hBM-MSCs. The K562 cells cultured alone served as negative control. Briefly, at the end of the co-culture period, the K562 cells were carefully removed and separated from the adherent MSCs. The K562 cells were carefully washed thrice with $2 \mathrm{ml}$ of phosphate-buffered saline (PBS) to remove any residues from the culture medium. The cells were processed for primary fixation where the cells were incubated in $4 \%$ glutaraldehyde solution for 6 hours at $4^{\circ} \mathrm{C}$. Following this, the cells were recovered by centrifugation (1500 rpm for $5 \mathrm{~min}$ ). Once the fixative solution was removed, $30 \mu \mathrm{l}$ of donkey serum was 
added to the cells and the cells were left at room temperature for 30 minutes to allow the mixture to solidify.

The solidified samples were cut into $1 \mathrm{~mm} 3$ slices, which were left in $4 \%$ glutaraldehyde solution for 2 hours at $4^{\circ} \mathrm{C}$. Once the fixative agent was removed, the samples were washed thrice with $0.1 \mathrm{M}$ sodium cacodylate buffer. Post fixation on the solidified samples was performed by adding $1 \%$ buffered osmium tetroxide and the samples were kept at $4^{\circ} \mathrm{C}$ for 2 hours. Following this, the samples washed thrice with $0.1 \mathrm{M}$ sodium cacodylate buffer. Then the samples were subjected to serial dehydration using increasing concentrations of acetone (35\%, 50\%, 75\%, 95\% and 100\%). The samples were then subjected to infiltration using 1:1 acetone/resin mixture, 1:3 acetone/resin mixture and 100\% resin, before they were embedded in $100 \%$ resin and allowed to polymerize in an oven set at $60^{\circ} \mathrm{C}$ for 24 to 48 hours. The embedded samples were cut into ultra-thin sections before the sections were viewed under the TEM (Hitachi, Japan) located at the Electron Microscopy Unit, Institute of Biological Science, University Putra Malaysia (UPM), Serdang, Malaysia.

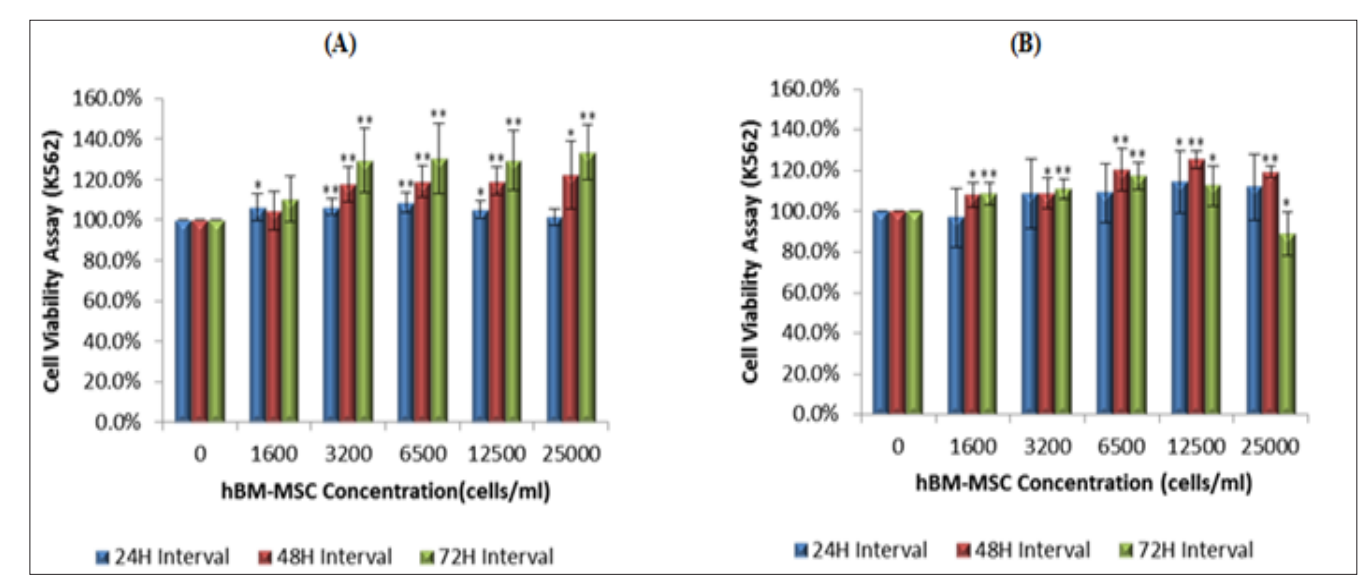

Figure 1: Dose- and time-dependent enhancement of the viability of K562 cell by hBM-MSC in direct contact co-culture using (a) complete medium and (b) serum-free medium. Viability of K562 cells at three time intervals A) 24 hour B) 48 hour and C) 72 hour and with increasing concentration of hBM-MSCs $(1600,3200,6500,12500,25000$ cells/ml) cultured in normal medium containing 10\% FBS was determined. Viability of K562 cells at three time intervals D) 24 hour E) 48 hour and F) 72 hour and with increasing concentration of hBM-MSCs $(1600,3200,6500,12500,25000$ cells/ml) cultured in specialized serum-free medium was determined. K562 cells cultured alone were used as control.

( ${ }^{*} \mathrm{P} \leq 0.05$ versus control; ${ }^{* *} \mathrm{P} \leq 0.05$ versus control)

\section{Results}

\section{Viability of $\mathrm{K} 562$ Cells Cultured in Direct Contact with hBM-MSCs}

The effects of hBM-MSC on K562 cells cultured in direct contact with the hBM-MSCs in the presence of complete medium enhanced the viability of the K562 cells in a dose- and time-dependent fashion (Figure $1 \mathrm{~A})$. The earliest significant $(\mathrm{p} \leq 0.05)$ increase in the viability of the K562 cells was $106.2 \% \pm 6.3 \%$ when 1600 cells $/ \mathrm{mL}$ hBM-MSC after 24 hours of culture. The highest viability $(\mathrm{p} \leq 0.001)$ was observed after 72 hours of co-culture $(133.6 \% \pm 13.7 \%)$. The same pattern was observed when the experiment was conducted using serum-free medium (Figure 1B). The results obtained were comparable with that obtained with direct contact culture experiments using complete medium. The earliest significant $(\mathrm{p} \leq 0.05)$ increase in the viability K562 cells $(114.2 \% \pm 15.3 \%)$ when these cells were co-cultured with 12500 cells/ml of the hBM-MSC for 24 hours. The highest cell viability $(125.4 \% \pm 4.4 \%$; $\mathrm{p} \leq 0.001)$ was observed when the K562 cells were co-cultured with 12500 cells/ml of the hBM-MSC for 48 hours. Unlike the direct contact culture (Figure 1A), there was a sharp drop in K562 cell viability towards the end of the culture experiment using specialised serumfree medium (Figure 1B). After 72 hours of co-culture, at the highest concentration ( 25000 cells $/ \mathrm{ml}$ ) of the hBM-MSC, the viability of the K562 cells decreased $(89.0 \% \pm 10.8 \% ; \mathrm{p} \leq 0.05)$.

\section{Viability of K562 Cells Cultured in Conditioned Medium}

When the K562 cells were cultured with conditioned medium harvested from the hBM-MSC, there was a marked drop $(87.7 \% \pm$ $6.8 \%$; $\leq 0.001$ ) in the viability of the K562 cells cultured for 24 hours in conditioned medium harvested from the hBM-MSC cultured for 72 hours (Figure 2A). However, the viability of K562 cells in all three conditions $(24,48$ and 72 hours conditioned medium) increased with highest viability (106.6\% $\pm 3.5 \%$; $\leq 0.001)$ observed following 48 hours of culture in the conditioned medium harvested from the hBM-MSC previously conditioned for 24 hours. The enhanced viability of the $\mathrm{K} 562$ cells remained elevated when these cells were cultured up to 72 hours for most of the culture conditions. A slight $\operatorname{dip}(100.5 \% \pm 6.1 \%$; $\mathrm{p} \geq 0.05)$ in $\mathrm{K} 562$ cell viability was observed in the 72 hour interval using the conditioned medium harvested from the hBM-MSC previously conditioned for 72 hours (Figure 2A). A similar pattern was observed when the experiment was conducted using serum-free medium (Figure 2B). As shown Figure 2B, there was a marked drop $(83.1 \% \pm 8.8 \%$; $\mathrm{p} \leq 0.05)$ in the viability of the K562 cells cultured for 24 hours in serum-free conditioned medium harvested from the hBM-MSC cultured for 72 hours. However, the 
viability of K562 cells in all three conditions (24, 48 and 72 hours serum-free conditioned medium) increased with highest viability (115.4\% $\pm 7.8 \% ; \mathrm{p} \leq 0.001)$ observed following 48 hours of culture in the conditioned medium harvested from the hBM-MSC cultured for 48 hours. The enhanced viability of K562 cells was sustained towards the end of the culture experiment.

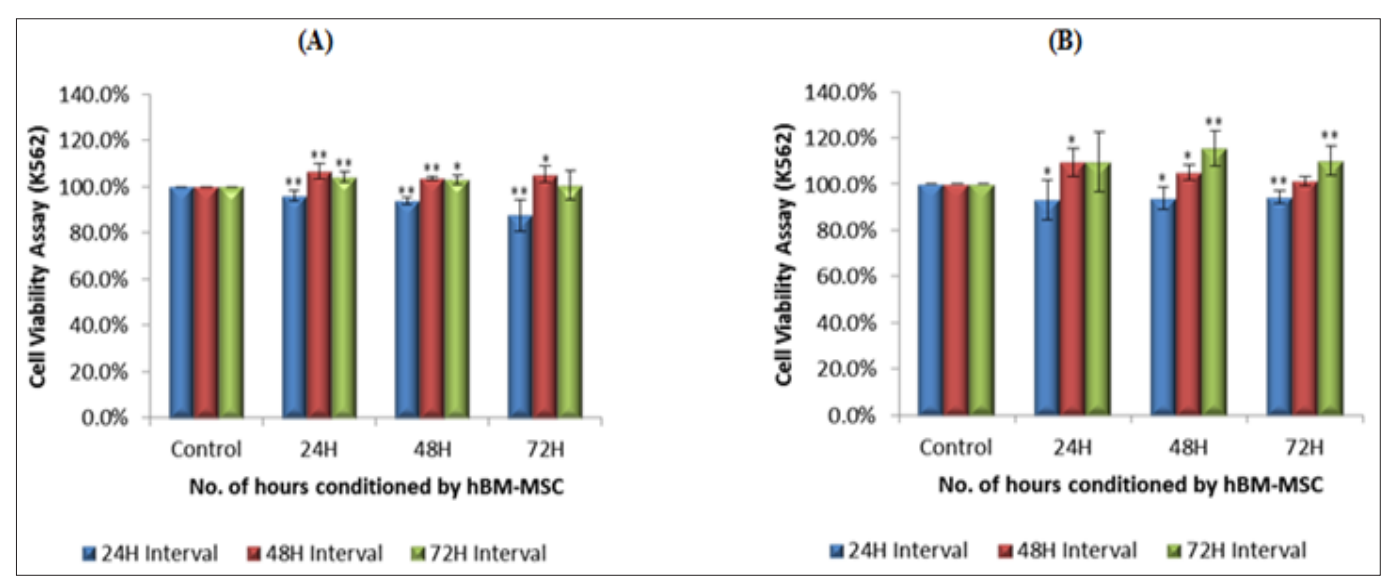

Figure 2: Dose- and time-dependent enhancement of the viability of K562 cell cultured using conditioned medium obtained using (a) complete medium and (b) serum-free medium. Viability of K562 cells at three time intervals A) 24 hour B) 48 hour and C) 72 hour and with increasing concentration of hBM-MSCs (1600, 3200, 6500, 12500, 25000 cells/ml) cultured in normal medium containing 10\% FBS was determined. Viability of K562 cells at three time intervals D) 24 hour E) 48 hour and F) 72 hour and with increasing concentration of hBM-MSCs (1600, 3200, 6500, 12500, 25000 cells/ml) cultured in specialized serumfree medium was determined. K562 cells cultured alone were used as control.

( ${ }^{*} \mathrm{P} \leq 0.05$ versus control; ** $\mathrm{P} \leq 0.05$ versus control).

\section{Viability of K562 cells Cultured in Transwell (non- contact) with hBM-MSCs}

The effects of hBM-MSC on the viability of K562 cells physically separated by Transwell inserts with two pore sizes $(0.4 \mu \mathrm{m}$ or 1.0 $\mu \mathrm{m}$ ) were investigated as described earlier. As shown in Figure 3A, the viability of the K562 cultured using the Transwell with $0.4 \mu \mathrm{m}$ pore size was found to be lower than the control, irrespective of the hBM-MSC concentration used and the culture duration. The lowest cell viability was found to be $90.1 \% \pm 3.8 \%(p \leq 0.001)$ when the
K562 cells were cultured with 12500 cells/ml of the hBM-MSC for 24 hours. The viability of the K562 cultured using the Transwell with $1.0 \mu \mathrm{m}$ pore size was also found to be lower than the control, irrespective of the hBM-MSC concentration used for the 24 and 48 hour interval (Figure 3B). The lowest cell viability was found to be $90.6 \% \pm 6.0 \%(\mathrm{p} \leq 0.05)$, when the K562 cells were cultured with 25000 cells $/ \mathrm{ml}$ of the hBM-MSC for 24 hours. However, at the 72 hour interval, K562 cell viability was found to be higher than the control, irrespective of the hBM-MSC concentration used. Highest cell viability was found to be $111.9 \% \pm 3.1 \%$ ( $\mathrm{p} \leq 0.001)$.

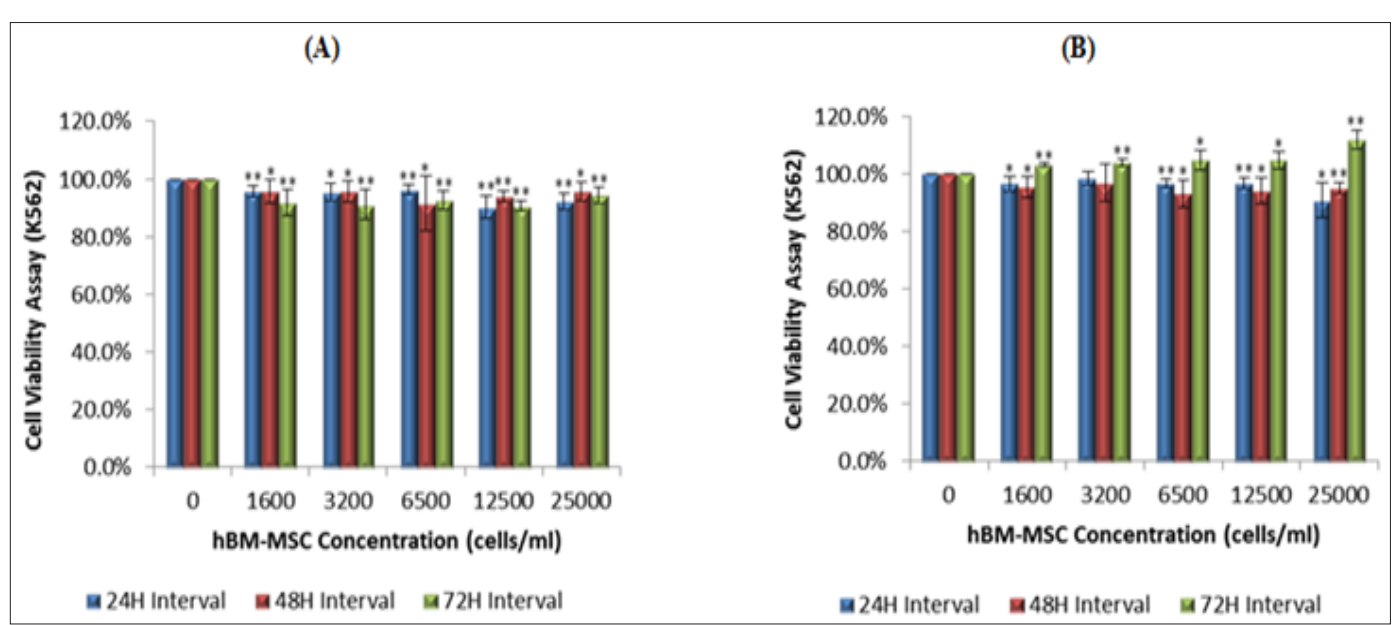

Figure 3: Dose- and time-dependent enhancement of the viability of K562 cell by hBM-MSC in Transwell (non-contact) inserts of (a) (a) $0.4 \mu \mathrm{m}$ and (b) $1.0 \mu \mathrm{m}$ pore size cultured using complete medium. Viability of K562 cells at three time intervals A) 24 hour B) 48 hour and C) 72 hour and with increasing concentration of hBM-MSCs (1600, 3200, 6500, 12500, 25000 cells/ml) cultured in Transwell inserts determined using the MTT assay. K562 cells cultured alone were used as control (0). ( ${ }^{*} \mathrm{P} \leq 0.05$ versus control; ** $\mathrm{P} \leq 0.05$ versus control) 


\section{Human Bone Marrow-Derived Mesenchymal Stem Cells (hBM-MSCs) Leads to an Increase in Mitochondria in K562 Cells}

Transmission electron microscopy was performed to investigate for any ultrastructural changes in K562 cells associated with cocultured with hBM-MSCs that could be appreciated under the transmission electron microscope. As shown in Figure 4, K562 cells co-cultured with hBM-MSCs in both complete medium containing $10 \%$ FBS as well as in specialised serum-free medium are shown to express higher levels of mitochondrial masses as compared to K562 cells cultured alone after co-culture duration of 24 hours. The increase in mitochondrial masses was shown to be sustained in the 48 hour interval, as shown in Figure 4(ii). In the 72 hour interval, mitochondrial masses was still expressed significantly higher in K562 cells co-cultured with hBM-MSCs in complete medium containing 10\% FBS than in K562 cells cultured alone. However, as compared to the 48 hour interval, K562 cells co-cultured with hBM-MSCs in serum-free medium showed less expression of mitochondrial masses at the 72 hour interval.

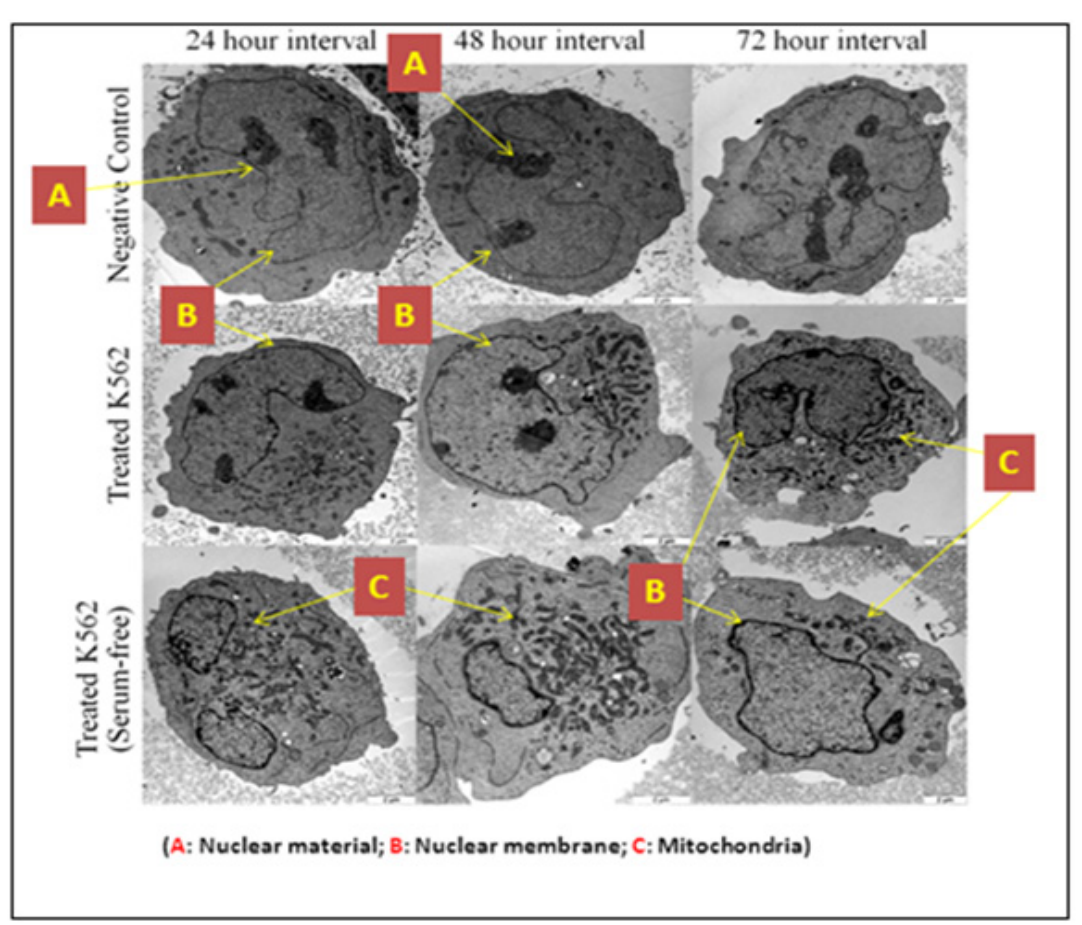

Figure 4: The morphology of the K562 cells co-cultured with the hBM-MSCs was observed using transmission electron microscopy (TEM) after (i) 24 hours, (ii) 48 hours or (iii) 48 hours of co-culture. The TEM pictures show morphology of K562 cells alone, K562 cells co-cultured with hBM-MSCs in complete medium and/or serum-free.

\section{Discussion}

In this study, hBM-MSCs are found to increase viability of K562 cells in both normal culture medium with $10 \%$ FBS and specialized culture medium without serum. This finding is supported by several studies, documenting that hBM-MSCs provide pro-survival signals to cancer $[1,25]$. There have also been several studies which demonstrated that hBM-MSCs increase the survival of other subtypes of leukemic cells (20-23). Amongst these studies, some reported that hBM-MSCs require direct cell-to-cell surface interactions to efficiently exert the aforementioned effects [21,23]. In these culture experiments, the effects of hBM-MSCs on the viability of K562 cells were quantified using 3-(4, 5-Dimethylthiazol2-yl)-2,5-diphenyltetrazolium bromide (MTT). In viable cells, the yellow tetrazole would be reduced to purple formazan crystals. Dissolution of the crystals using a solvent provides a colorimetric endpoint which when quantified in a microplate reader, has a positive correlation with the viability of the cells. Due to this property, MTT assay has been widely used to evaluate viability of various types of cells [19].
The increase in viability of K562 cells shown in the Transwell (1.0 $\mu \mathrm{m}$ pore size) experiments (Figure $3 \mathrm{~B}$ ) suggests that hBMMSCs may be producing soluble factor(s) of sizes smaller than 1.0 $\mu \mathrm{m}$, which may affect the viability of the K562 cells. As there was no increase in viability of K562 cells observed in the Transwell ( $0.4 \mu \mathrm{m}$ pore size) experiments (Figure $3 \mathrm{~A}$ ), it can be inferred that the soluble factor(s) involved may be bigger than $0.4 \mu \mathrm{m}$ in size. The hBM-MSCs have been documented to secrete a myriad of various chemicals, including PGE2 [24], IL-1囚 [25], TGF- $\bigotimes[26]$ and leukemia inhibitory factor (LIF) [27]. The findings observed in this study could be attributed to the possible involvement of soluble factor, stromal cell-derived factor-1 (SDF-1/CXCL12) in the interactions between hBM-MSCs and leukemia, which has been previously reported [28].

The results from the conditioned medium experiments (Figures 2A \& 2B) also showed an increase in the viability of K562 cells in medium previously conditioned by hBM-MSCs. These results suggest that hBM-MSCs may produce factors as discussed earlier, independent of any interactions with K562 cells. The hBM-MSCs 
may plausibly secrete those soluble factors constitutively. This finding has been previously reported [29]. The increase in viability of K562 cells seen through the interactions with hBM-MSCs implies that hBM-MSCs may be contributing to the survival and sustenance of the malignant process. It may account for the increase in recurrence rate of leukemia previously reported in leukemic patients receiving MSC therapy in conjunction with bone marrow transplant [30]. It is known that MSCs are currently explored as potential therapeutic agent for various pathological diseases. Given that MSCs may contribute to oncogenesis, it is imperative for us to proceed with caution in our utilization of MSCs as therapeutic option before we fully understand the nature of MSCs as well as their interactions within the human body.

Furthermore, the data obtained from this study suggests that the time duration and concentration in which K562 cells are cocultured with hBM-MSCs should be considered. For instance, hBM-MSC concentrations as low as 1600 cells/mL have been able to substantially increase the viability of K562 cells. Given the timeand dose-dependent relationship between K562 cells and hBMMSCs, it is plausible to hypothesize that the biological effect hBMMSCs may have on K562 cells in an in vivo setting can be immense. Yet, it should also be noted that there have been conflicting findings regarding the effects of hBM-MSCs on leukemic cells. Several studies have reported that hBM-MSCs possess anti-proliferative properties on various subtypes of leukemia [16,31]. The heterogeneity and source of the hBM-MSCs may also account for differing results in the different studies.

Under the transmission electron microscope, an increase in mitochondrial masses in K562 cells co-cultured with hBM-MSCs can be appreciated in the first 24-hour interval. Since mitochondrial elements are responsible for the reduction of MTT and formation of purple formazan crystals [32], the increase in mitochondrial masses correlate with the increased reading obtained in the MTT viability assays performed. Although K562 cells have been viewed under the transmission electron microscope for various other studies [33], this study represents a novel attempt to observe for any ultrastructural changes that could be appreciated through the interaction between cell entities. As there has not been any documentation detailing the ultrastructural effects hBM-MSCs have on $\mathrm{K} 562$ cells, the mechanistic nature of how the number of mitochondria was elevated remains to be elucidated [34].

\section{Conclusion}

In conclusion, our results demonstrated that hBM-MSC indeed possess a modulatory effect on the cellular viability of K562 leukemic cells. For the modulatory effect to take place effectively, cell-to-cell interaction looks to be essential. Through our Transwell experiments, our data also suggests that hBM-MSC may also secrete soluble factor(s) that exert a similar effect on K562 cell viability. Therefore, these results will support the notion that hBM-MSCs exert their modulatory effect on K562 cell viability via at least two pathways. Our research has also demonstrated significant ultrastructural changes in K562 leukemic cells through interaction with hBM-MSCs. Although the mechanisms in which hBM-MSCs exert their effect on K562 cell viability remains to be elucidated, the increase in mitochondrial mass demonstrated in our TEM has provided a valuable clue for future works.

\section{Acknowledgement and Role of Funding Source}

The International Medical University, Malaysia funded this study [grant number: BMSc1-01/2011(10)] and provided all required facilities. Cytopeutics (Malaysia) provided the human bone marrow-derived mesenchymal stem cells (hBM-MSCs) used in this study. The electron microscopy work was carried out the TEM Unit, Institute of Biological Sciences, UPM. The funding source had no involvement in the study design; collection, analysis and interpretation of data; writing of report; and in the decision to submit the paper for publication.

\section{References}

1. Hajdu S (2003) A note from history: The discovery of blood cells. Annals of Clinical and Laboratory Science 33(2): 237-238.

2. Estey E, Döhner H (2006) Acute myeloid leukaemia. The Lancet 368(9550): 1894-1907.

3. Ferlay J, Shin HR, Bray F, Forman D, Mathers C, et al. (2010) Estimates of worldwide burden of cancer in 2008: GLOBOCAN 2008. International Journal of Cancer 127(12): 2893-2917.

4. Friedenstein A J, Petrakova KV, Kurolesova AI, Frolova GP (1968) Heterotopic transplants of bone marrow. Transplantation 6(2): 230-247.

5. Ma T (2010) Mesenchymal stem cells: From bench to bedside. World Journal of Stem Cells 2(2): 13-17.

6. Nauta AJ, Fibbe WE (2007) Immunomodulatory properties of mesenchymal stromal cells. Blood 110(10): 3499-3506.

7. Barker JN, Wagner JE (2003) Umbilical-cord blood transplantation for the treatment of cancer. Nature Reviews Cancer 3(7): 526-532.

8. Zhou H, Guo M, Bian C, Sun Z, Yang Z, et al. (2010) Efficacy of bone marrowderived mesenchymal stem cells in the treatment of sclerodermatous chronic graft-versus-host disease: clinical report. Biology of Blood and Marrow Transplantation 16(3): 403-412.

9. Kebriaei P, Isola L, Bahceci E, Holland K, Rowley S, et al. (2009) Adult human mesenchymal stem cells added to corticosteroid therapy for the treatment of acute graft-versus-host disease. Biol Blood Marrow Transplant 15(7): 804-811.

10. Le Blanc K, Frassoni F, Ball L, Locatelli F, Roelofs H, et al. (2008) Developmental Committee of the European Group for Blood and Marrow Transplantation: Mesenchymal stem cells for treatment of steroidresistant, severe, acute graft-versus-host disease: a phase II studies. Lancet 371(9624): 1579-1586.

11. Von Bonin M, Stölzel F, Goedecke A, Richter K, Wuschek N, et al. (2009) Treatment of refractory acute GVHD with third-party MSC expanded in platelet lysate-containing medium. Bone Marrow Transplant 43(3): 245-251.

12. Li L, Tian H, Yue W, Zhu F, Li S, et al. (2011) Human mesenchymal stem cells play a dual role on tumor cell growth in vitro and in vivo. Journal of Cellular Physiology 226(7): 1860-1867.

13. Liu S, Ginestier C, Ou SJ, Clouthier SG, Patel SH, et al. (2011) Breast cancer stem cells are regulated by mesenchymal stem cells through cytokine networks. Cancer Research 71(2): 614-624.

14. Loebinger MR, Eddaoudi A, Davies D, Janes SM (2009) Mesenchymal stem cell delivery of TRAIL can eliminate metastatic cancer. Cancer Research 69(10): 4134-4142.

15. Garrido SM, Appelbaum FR, Willman CL, Banker DE (2001) Acute myeloid leukemia cells are protected from spontaneous and druginduced apoptosis by direct contact with a human bone marrow stromal cell line (HS-5). Experimental Hematology 29(4): 448-457. 
16. Mudry RE, Fortney JE, York T, Hall BM, Gibson LF (2000) Stromal cells regulate survival of B-lineage leukemic cells during chemotherapy. Blood 96(5): 1926-1932.

17. Klopp AH, Lacerda A, Gupta A, Debeb BG, Solley T, Li et al. (2010) Mesenchymal stem cells promote mammosphere formation and decrease E-cadherin in normal and malignant breast cells. Plos ONE 5(8): e12180.

18. Wei Z, Chen N, Guo H, Wang X, Xu F, et al. (2009) Bone marrow mesenchymal stem cells from leukemia patients inhibit growth and apoptosis in serum-deprived K562 cells. J Exp Clin Cancer Res 28(1): 141.

19. Mosmann T (1983) Rapid colorimetric assay for cellular growth and survival: application to proliferation and cytotoxicity assays. J Immunol Methods 65(1-2): 55-63.

20. Jin L, Tabe Y, Konoplev S, Xu Y, Leysath CE, et al. (2008) CXCR4 upregulation by imatinib induces chronic myelogenous leukemia (CML) cell migration to bone marrow stroma and promotes survival of quiescent CML cells. Molecular Cancer Therapeutics 7(1): 48-58.

21. Iwamoto S, Mihara K, Downing JR, Pui CH, Campana D (2007) Mesenchymal cells regulate the response of acute lymphoblastic leukemia cells to asparaginase. Journal of Clinical Investigation 117(4): 1049-1057.

22. Lagneaux L, Delforge A, Bron D, De Bruyn C, Stryckmans P (1998) Chronic lymphocytic leukemic B cells but not normal B cells are rescued from apoptosis by contact with normal bone marrow stromal cells. Blood 91(7): 2387-2396.

23. Manabe A, Coustan Smith E, Behm FG, Raimondi SC, Campana D (1992) Bone marrow-derived stromal cells prevent apoptotic cell death in B-lineage acute lymphoblastic leukemia. Blood 79(9): 2370-2377.

24. Tse WT, Pendleton JD, Beyer WM, Egalka MC, Guinan EC (2003) Suppression of allogeneic T-cell proliferation by human marrow stromal cells: implications in transplantation. Transplantation-Baltimore 75(3): 389-397.
25. Groh ME, Maitra B, Szekely E, Koç ON (2005) Human mesenchymal stem cells require monocyte-mediated activation to suppress alloreactive $\mathrm{T}$ cells. Experimental Hematology 33(8): 928-934.

26. Di Nicola M, Carlo-Stella C, Magni M, Milanesi M, Longoni PD, et al. (2002) Human bone marrow stromal cells suppress T lymphocyte proliferation induced by cellular or nonspecific mitogenic stimuli. Blood 99: 38383843.

27. Nasef A, Mathieu N, Chapel A, Frick J, Francois S, et al. (2007) Immunosuppressive effects of mesenchymal stem cells: involvement of HLA-G. Transplantation 84(2): 231-237.

28. Burger JA, Kipps TJ (2006) CXCR4: a key receptor in the crosstalk between tumor cells and their microenvironment. Blood 107(5): 17611767.

29. Konopleva M, Konoplev S, Hu W, Zaritskey AY, Afanasiev BV, et al. (2002) Stromal cells prevent apoptosis of AML cells by up-regulation of antiapoptotic proteins. Leukemia 16(9): 1713-1724.

30. Ning H, Yang F, Jiang M, Hu L, Feng K, Zhang, et al. (2008) The correlation between cotransplantation of mesenchymal stem cells and higher recurrence rate in haematologic malignancy patients: outcome of a pilot clinical study. Leukaemia 22(3): 593-599.

31. Ramasamy R, Lam EW, Soeiro I, Tisato V, Bonnet D, et al. (2006) Mesenchymal stem cells inhibit proliferation and apoptosis of tumor cells: impact on in vivo tumor growth. Leukemia 21(2): 304-310.

32. Bernas T, Dobrucki J (2002) Mitochondrial and nonmitochondrial reduction of MTT: Interaction of MTT with TMRE, JC-1, and NAO mitochondrial fluorescent probes. Cytometry 47(4): 236-242.

33. Bobichon H, Okiemi MG, Broglio C, Ploton D, Dufer J (1992) Adriamycin resistance is characterized by ultrastructural changes in human leukaemic K562 cells in vitro. Biology of the Cell 74(3): 287-297.

34. Noseda A, White JG, Godwin PL, Jerome WG, Modest EJ (1989) Membrane damage in leukemic cells induced by ether and ester lipids: an electron microscopic study. Exp Mol Pathol 50(1): 69-83.
This work is licensed under Creative Commons Attribution 4.0 License

Submission Link: https://biomedres.us/submit-manuscript.php

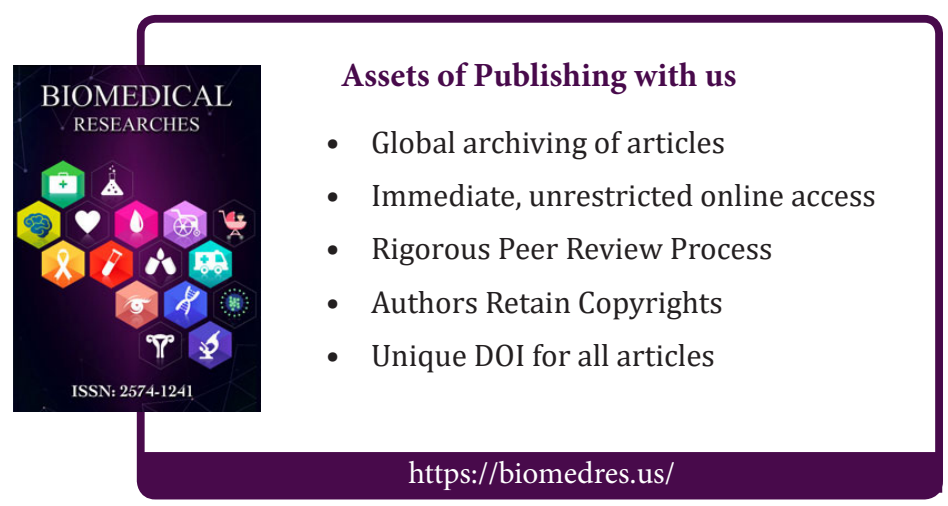

